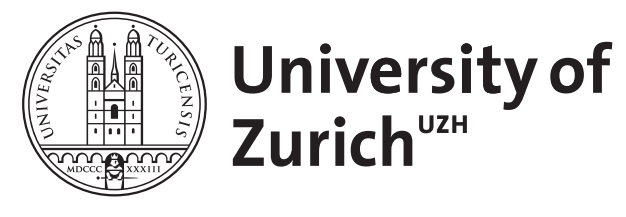

\title{
Existence and multiplicity result for a class of second order elliptic equations
}

\author{
Hofer, Helmut
}

\begin{abstract}
SynopsisSome second order semilinear elliptic boundary value problems of the AmbrosettiProdi-type are studied. Existence and multiplicity of solutions is proved in dependence on a parameter. Constructing a global strongly increasing fixed point operator in a suitable function space, observing under appropriate conditions, which are in some sense optimal-that the fixed point operator has some properties similar to a strongly positive linear endomorphism, one unifies and improves the treatment of such problems, whether the nonlinearity is dependent on the gradient or not, and obtains some new results.
\end{abstract}

DOI: https://doi.org/10.1017/s0308210500017303

Posted at the Zurich Open Repository and Archive, University of Zurich ZORA URL: https://doi.org/10.5167/uzh-154925

Journal Article

Published Version

Originally published at:

Hofer, Helmut (1981). Existence and multiplicity result for a class of second order elliptic equations. Proceedings of the Royal Society of Edinburgh Section A: Mathematics, 88(1-2):83-92.

DOI: https://doi.org/10.1017/s0308210500017303 


\section{Existence and multiplicity result for a class of second order elliptic equations}

\section{Helmut Hofer}

Mathematics Institute, University of Zurich, Freiestrasse 36, CH-8032 Zurich, Switzerland

(MS received 23 January 1980. Revised MS received 30 June 1980)

\section{Synopsis}

Some second order semilinear elliptic boundary value problems of the Ambrosetti-Prodi-type are studied. Existence and multiplicity of solutions is proved in dependence on a parameter. Constructing a global strongly increasing fixed point operator in a suitable function space, observing-under appropriate conditions, which are in some sense optimal-that the fixed point operator has some properties similar to a strongly positive linear endomorphism, one unifies and improves the treatment of such problems, whether the nonlinearity is dependent on the gradient or not, and obtains some new results.

\section{Introduction and statement of the results}

In this paper we study the semilinear boundary value problem (BVP)

$$
\begin{aligned}
& L u=G(x, u, D u)+\operatorname{tr} \text { in } \Omega \\
& B u=0 \text { on } \partial \Omega,
\end{aligned}
$$

where $t$ is a real parameter value. Here $\Omega$ denotes a bounded domain in $\mathbb{R}^{n}$ whose boundary $\partial \Omega$ is a $C^{2}$-submanifold of dimension $n-1$ such that $\Omega$ lies locally on one side of $\partial \Omega$. By $L$ we denote the strongly uniformly elliptic differential operator $L u=-\sum_{i, i=1}^{n} a_{i j} D_{i} D_{j} u+\sum_{i=1}^{n} a_{i} D_{i} u+a_{0} u$, where $a_{i j}=a_{i j}, a_{i}, a_{0} \in C(\bar{\Omega})$, and, adding $\alpha I, \alpha>0$, to both sides of $\left(\mathrm{P}_{t}\right), a_{0}(x) \geqq \varepsilon>0$ for all $x \in \bar{\Omega}$. B denotes either the Dirichlet boundary operator, or the operator $B u=\partial u / \partial \nu+c_{0} u$, where $\nu \in C^{1}\left(\partial \Omega, \mathbb{R}^{n}\right)$ is an outward pointing, nowhere tangent vectorfield on $\partial \Omega$ and $c_{0} \in C^{1}(\partial \Omega, \mathbb{R}), c_{0} \geqq 0$. Moreover

$$
r \in C(\bar{\Omega}) \backslash\{0\} \text { satisfies } r(x) \geqq 0 \text { in } \Omega \text {. }
$$

Let $\lambda_{1}$ denote the principal eigenvalue of the linear BVP $L u=\lambda u$ in $\Omega, B u=0$ on $\partial \Omega$. Since $a_{0} \geqq \varepsilon$ we have $\lambda_{1}>0$.

For the nonlinearity $G$ we assume:

(G1) $G: \Delta \equiv \bar{\Omega} \times \mathbb{R} \times \mathbb{R}^{n} \rightarrow \mathbb{R}$ is continuous, continuously differentiable with respect to $s$ and $\xi$ and verifies the growth conditions

$$
\begin{array}{rll}
|G(x, s, \xi)| \leqq c_{1}(|s|)\left(1+|\xi|^{2}\right) & \text { for all } & (x, s, \xi) \in \Delta \\
|G(x, s, \xi)| \leqq c_{2}\left(s^{+}\right)(1+|s|+|\xi|) & \text { for all } & (x, s, \xi) \in \Omega^{0} \times \mathbb{R} \times \mathbb{R}^{n}
\end{array}
$$

where $c_{i}: \mathbb{R}^{+} \rightarrow \mathbb{R}^{+}$are suitable increasing functions and $\Omega^{0}$ denotes the set $\{x \in \Omega \mid r(x)=0\}$ and $s^{+}=s$ if $s \geqq 0$ and $s^{+}=0$ otherwise. 
(G2) There exists a continuous function $G^{*}: \bar{\Omega} \times \mathbb{R} \rightarrow \mathbb{R}$ such that $G(x, s, \xi) \geqq$ $G^{*}(x, s)$ for all $(x, s, \xi) \in \Delta$ and

$$
\begin{aligned}
& \limsup _{s \rightarrow-\infty} G^{*}(x, s) / s<\lambda_{1} \\
& \liminf _{s \rightarrow+\infty} G^{*}(x, s) / s>\lambda_{1}
\end{aligned}
$$

uniformly for $x \in \bar{\Omega}$.

Our main results are:

THEOREM 1. Assume $r$ and $G$ satisfy (1) and (G1), (G2), respectively. Then there exists a $t_{0} \in \mathbb{R}$ such that $\left(\mathrm{P}_{t}^{*}\right)$ is solvable for $t<t_{0}$ and not solvable for $t>t_{0}$.

THEOREM 2. Assume the hypotheses of Theorem 1 are satisfied and in addition there exists for all bounded intervals $\Gamma \subset \mathbb{R}$ a constant $M=M(\Gamma)$ such that $u_{t}(x) \leqq M$ for all $x \in \bar{\Omega}$, where $u_{t}$ is an arbitrary solution of $\left(\mathrm{P}_{t}\right), t \in \Gamma$. Then there is $a t_{0} \in \mathbb{R}$ such that $\left(\mathrm{P}_{\mathrm{t}}\right)$ possesses at least two solutions for $t<t_{0}$, at least one solution for $t=t_{0}$ and no solution for $t>t_{0}$.

As an application we have:

COROLlary. If $r$ and $G$ satisfy (1) and (G1), (G2), respectively, and in addition

(G3) for all $m \in \mathbb{R}$ there exists a constant $M=M(m)$ such that

$$
|G(x, s, \xi)| \leqq M(1+|s|+|\xi|)
$$

for all $s \geqq m, x \in \bar{\Omega}$ and $\xi \in \mathbb{R}^{n}$, then the hypotheses of Theorem 2 are satisfied.

Some remarks concerning the comparison of our results with related former research are in order.

1. If $r(x)>0$ in $\Omega$ and $B u=u$ we obtain a recent result of Kazdan and Kramer [1]. They prove a theorem of the type of Theorem 1 via the method of sub-and supersolutions. One should also mention the paper of Kazdan and Warner [5] in which similar problems for the case where $G$ is not depending on the gradient have been studied. If $r(x) \geqq 0$ in $\Omega$ but $r \neq 0$, the basic step in [1] and [5], the construction of a supersolution for a suitable parameter value $t$ does not carry over to our situation. Moreover no multiplicity result is obtained there and no assertion is made for $t=t_{0}$. Note however that for the multiplicity result we need an additional a priori estimate.

2. If $r \supsetneqq 0$ we obtain an extension and sharpening of a result of Hess [2] where the nonlinearity was independent of the gradient. The technique developed in [2] does not apply to our situation if one has gradient-dependence.

3. In a recent paper Amann and Hess [7] proved a theorem of the type of Theorem 2 for the nonlinear BVP $L u=f(x, u, t)$ in $\Omega, B u=0$ on $\partial \Omega$, where $t$ denotes a real parameter value. If $G$ is independent of the gradient and $r(x)>0$ in $\Omega$ their result is applicable to our situation. Hence, Theorem 2 and the corollary extend their result in some respect to the case where $G$ is depending on the gradient and the function $r$ satisfies $r \supsetneqq 0$.

4. As a by-product we obtain a much simpler method for studying the equation $L u=G(x, u, D u)$ in $\Omega, B u=0$ on $\partial \Omega$, if sub- and supersolutions $\bar{u} \leqq \hat{u}$ are given, as in the paper of Amann and Crandall [4]. Their method only allowed the 
construction of an increasing fixed point operator on the order interval $[\bar{u}, \hat{u}]$. Our strongly increasing fixed point operator is a global one, which has some advantages, for example in studying multiplicity.

5. Questions similar to our problem have also been discussed by Dancer [8] for equations of the type $L u=g(u)-f$.

Acknowledgment. I wish to thank M. Crandall and P. Hess for helpful suggestions.

\section{A basic proposition}

Fix $p>n$ throughout this paper. We will use the standard notation for the function spaces and denote by $C_{B}^{1}(\bar{\Omega})$ and $W_{B}^{2 . p}(\Omega)$ the closed subspaces of $C^{1}(\bar{\Omega})$ and $W^{2, p}(\Omega)$ consisting of those functions which satisfy the boundary condition $B u=0$ on $\partial \Omega$. In the sequel we will need the following proposition.

Proposirion. Assume $\gamma: C^{1}(\bar{\Omega}) \rightarrow L^{\infty}(\Omega)$ is a continuous operator which satisfies $|\gamma(u)(x)| \leqq c(|u(x)|)\left(1+|D u(x)|^{2}\right)$ for all $x \in \bar{\Omega}$, where $c: \mathbb{R}^{+} \rightarrow \mathbb{R}^{+}$is a suitable increasing function and $\gamma(0)=0$. Moreover assume $\gamma$ admits for all $u, v \in C^{1}(\bar{\Omega})$ the representation

$$
\gamma(u)-\gamma(v)=\sum_{i=1}^{n} b_{i} D_{i}(u-v)+b_{0} \cdot(u-v)
$$

with $b_{i}=b_{i}(u, D u, v, D v) \in L^{\infty}(\Omega), i=0, \ldots, n$, and $b_{0} \geqq 0$. Then we have

(a) The problem

$$
L u+\gamma(u)=f \quad \text { in } \Omega, \quad B u=0 \quad \text { on } \partial \Omega,
$$

is for all $f \in L^{\infty}(\Omega)$ uniquely solvable in $W^{2, p}(\Omega)$ and the solution operator $H: L^{\infty}(\Omega) \rightarrow C_{\mathrm{B}}^{1}(\bar{\Omega})$ is strongly increasing and compact where the function spaces are equipped with the natural order structures. If $S \subset L^{\infty}(\Omega)$ is a $L^{\alpha}$-bounded set, equipped with the induced $L^{p}$-topology given by the imbedding $L^{\alpha}(\Omega) \rightarrow L^{p}(\Omega)$, then the operator $H_{S}: S \rightarrow C_{B}^{1}(\bar{\Omega}): f \rightarrow H f$ is continuous.

(b) If there exist an open subset $\Omega^{*} \subset \Omega$ and for all $v \in C^{1}(\bar{\Omega})$ a constant $M(v) \geqq 0$ such that $|\gamma(w+v)(x)-\gamma(v)(x)| \leqq M(v)(|w(x)|+|D w(x)|)$ for $x \in \Omega^{*}$ and $w \leqq 0$, and if $r$ satisfies (1) with $r(x)>0$ for all $x \in \Omega \backslash \Omega^{*}$, then for all $a \in C(\bar{\Omega})$ and all $\phi \in C_{B}^{1}(\bar{\Omega})$ we find $T=T(a, \phi, r) \in \mathbb{R}$ such that $H(a+t r) \leqq \phi$ for all $t \leqq T$.

Remark 1. Roughly speaking (b) says that linear growth of the operator $u \rightarrow \gamma(u)$ in $u$ and $|D u|$ on the set $\{x \in \Omega \mid r(x)=0\}$ implies that a solution of $(*)$ with $f=a+t r$ becomes negative if $t \in \mathbb{R}$ is small enough.

Remark 2. A typical example for such an operator is the following: Consider a continuous map $\beta: \bar{\Omega} \times \mathbb{R} \times \mathbb{R}^{n} \rightarrow \mathbb{R}:(x, s, \xi) \rightarrow \beta(x, s, \xi)$ such that $\partial \beta / \partial s$ and $\partial \beta / \partial \xi$ exist and are continuous, which satisfies the growth conditions (G1) (i) and (ii), and in addition let $\partial \beta / \partial s \geqq 0$. Define $\gamma: C^{1}(\bar{\Omega}) \rightarrow L^{\infty}(\Omega)$ by $\gamma(u)(x)=$ $\beta(x, u(x), D u(x))$. We find for $u, v \in C^{1}(\vec{\Omega})$

$$
\begin{aligned}
\gamma(u)-\gamma(v)= & \sum_{i=1}^{n}\left(\int_{0}^{1}\left(\partial \beta / \partial \xi_{i}\right)(., v+t(u-v), D v+t D(u-v)) d t\right)\left(D_{i} u-D_{i} v\right) \\
& +\left(\int_{0}^{1}(\partial \beta / \partial s)(., v+t(u-v), D v+t D(u-v)) d t\right)(u-v)
\end{aligned}
$$

which is an admissible representation. 
For the proof of the proposition we need the following lemmata:

LemMa 1. Suppose $b_{i} \in L^{\infty}(\Omega), i=0, ., ., n$, and $b_{0}(x) \geqq 0$. Let $u \in W^{2, p}(\Omega)$ satisfy the inequalities

$$
L u+\sum_{i=1}^{n} b_{i} D_{i} u+b_{0} u \geqq 0 \quad \text { in } \Omega, \quad B u \geqq 0 \quad \text { on } \partial \Omega .
$$

Then $u \geqq 0$. If $u \neq 0$ then $u(x)>0$ for all $x \in \Omega$ and if $u(x)=0$ for some $x \in \partial \Omega$, then $(\partial u / \partial \nu)(x)<0$ where $\nu$ is an outward pointing vector at $x$ which is not tangential.

Lemma 1 follows from Bony's maximum principle [6].

Lemma 2. For every $b \in L^{\infty}(\Omega)$ there is exactly one solution $u \in W^{2, p}(\Omega)$ of the problem $L u=b\left(1+|D u|^{2}\right)$ in $\Omega, B u=0$ on $\partial \Omega$. Moreover there is an increasing function $d: \mathbb{R}^{+} \rightarrow \mathbb{R}^{+}$such that $\|u\|_{2, p} \leqq d\left(\|b\|_{\infty}\right)$. The function $d$ is depending only on $L, \Omega, p$, and $n$.

The proof is a trivial variant of a result in [4].

Proof of the proposition. By the hypotheses on $L$ and $\gamma$ we find constants $M_{1}<0$ and $M_{2}>0$ such that the functions $\phi_{i}(x)=M_{i}, i=1,2$, are strict sub- and supersolutions of $(*)$. Let us denote by $A$ the order interval $\left[\phi_{1}, \phi_{2}\right]$ in $C^{1}(\bar{\Omega})$. Assume $u, v \in W^{2, p}(\Omega)$ satisfy

$$
L u+\gamma(u) \geqq L v+\gamma(v) \quad \text { in } \Omega, \quad B u \geqq B v \quad \text { on } \partial \Omega .
$$

Using our representation of $\gamma$ and Lemma 1 we immediately find that a solution of $(*)$ is unique and that the solution operator - if it exists - is strongly increasing. Moreover, the solution of $(*)$ has to be in the interior of $A$ due to the fact that $\phi_{1}, \phi_{2}$ are strict sub- and supersolutions. Using Lemma 2 we obtain an $a$ priori estimate for the solution $u$. In fact, the solution $u$ of $(*)$ satisfies

$$
\begin{aligned}
& L u=b\left(1+|D u|^{2}\right) \quad \text { in } \Omega \\
& B u=0 \text { on } \partial \Omega,
\end{aligned}
$$

where $b=(-\gamma(u)+f)\left(1+|D u|^{2}\right)^{-1}$. Since $u$ has to be in int $(A)$ we find by the growth condition of $\gamma$ that $\|b\|_{L^{\infty}(\Omega)} \leqq$ const where the constant is only depending on $\phi_{1}$ and $\phi_{2}$. This implies by Lemma $2:\|u\|_{C^{\prime}(\bar{\Omega})} \leqq$ const. Let $B_{R}$ denote the open ball in $C^{1}(\bar{\Omega})$ with radius $R$ and let $A_{R}=A \cap B_{R}$. By our preceding discussion one immediately obtains for $R$ large enough that $\operatorname{deg}\left(I-P, A_{R}, 0\right)=1$, where $P: A_{R} \rightarrow C^{1}(\bar{\Omega})$ is defined by $P u=K(f-\gamma(u))$ and $K$ denotes the solution operator of the linear BVP $L u=g$ in $\Omega, B u=0$ on $\partial \Omega .\left(K: L^{\infty}(\Omega) \rightarrow C^{1}(\vec{\Omega})\right.$ compact). Up to now we have proved that $(*)$ is uniquely solvable for $f \in L^{\infty}(\Omega)$ and that the solution operator $H: L^{\infty}(\Omega) \rightarrow W_{B}^{2, p}(\Omega): f \rightarrow u$ is strongly increasing. Using Lemma 2 in the same way as before, we find that $H$ maps $L^{\infty}$-bounded sets into $W^{2, p}$-bounded sets. From the compactness of the imbedding $W_{B}^{2, p}(\Omega) \rightarrow$ $C_{B}^{1}(\bar{\Omega})$ we deduce that $H: L^{\infty}(\Omega) \rightarrow C_{B}^{1}(\bar{\Omega})$ maps bounded sets into relatively compact sets and moreover $H$ remains strongly increasing. Let $S \subset L^{\infty}(\Omega)$ be a $L^{\infty}$-bounded set equipped with the $L^{p}$-topology. Assume $H_{S}: S \rightarrow C_{B}^{1}(\bar{\Omega})$ is not continuous. Then there exists a sequence $\left(f_{n}\right) \subset S$ converging in $L^{\mathrm{p}}$ to some $f \in S$ such that $\left\|H f_{n}-H\right\|_{C^{\prime}(\bar{\Omega})} \geqq \delta$ for a suitable $\delta>0$. Since $\left(H f_{n}\right)$ is bounded in 
$W_{B}^{2, p}(\Omega)$ we may assume (for a subsequence) $H f_{n} \rightarrow u$ strongly in $C_{B}^{1}(\bar{\Omega})$. Taking the limit in

$$
L H f_{n}+\gamma\left(H f_{n}\right)=f_{n} \quad \text { in } \Omega, \quad B H f_{n}=0 \text { on } \partial \Omega
$$

we find

$$
L u+\gamma(u)=f \quad \text { in } \Omega, \quad B u=0 \quad \text { on } \partial \Omega .
$$

Hence, by the uniqueness of solutions we must have $u=H f$. This implies a contradiction: $0<\delta \leqq \lim \inf \left\|H f_{n}-H f\right\|_{C^{1}(\bar{\Omega})}=0$.

Now we prove (b). Let $a \in C(\bar{\Omega})$ and denote $H(a)$ by $v$. We have for $u=$ $H(a+t r)$ and $w=u-v$

$$
L w+\gamma(w+v)-\gamma(v)=t r \quad \text { in } \Omega, \quad B w=0 \quad \text { on } \partial \Omega .
$$

Let $\bar{\gamma}: C^{1}(\bar{\Omega}) \rightarrow L^{\infty}(\Omega): w \rightarrow \gamma(w+v)-\gamma(v)$ and denote by $\chi: \bar{\Omega} \rightarrow \mathbb{R}$ the characteristic function of $\Omega \backslash \Omega^{*}$. Define $\gamma^{*}: C^{1}(\bar{\Omega}) \rightarrow L^{\infty}(\Omega)$ by

$$
\gamma^{*}(w)(x)=(\chi \tilde{\gamma}(w))(x)+M(v) w(x)-M(v)|D w(x)| .
$$

Obviously $\gamma^{*}$ satisfies the hypotheses of part (a) and moreover we have for $w \leqq 0$

$$
\left(\tilde{\gamma}(w)-\gamma^{*}(w)\right)(x)=((1-\chi) \tilde{\gamma}(w))(x)-M(v) w(x)+M(v)|D w(x)| .
$$

If $x \in \Omega^{*}$ we deduce

$$
\begin{aligned}
\left(\tilde{\gamma}(w)-\gamma^{*}(w)\right)(x) & \geqq M(v)|w(x)|+M(v)|D w(x)|-|\bar{\gamma}(w)(x)| \\
& \geqq M(v)|w(x)|+M(v)|D w(x)|-M(v)|w(x)| \\
& \geqq 0 .
\end{aligned}
$$

If $x \in \Omega \backslash \Omega^{*}$ we find

$$
\left(\tilde{\gamma}(w)-\gamma^{*}(w)\right)(x)=M(v)|w(x)|+M(v)|D w(x)| \geqq 0 .
$$

Hence we have for all $w \in C^{1}(\bar{\Omega})$ with $w \leqq 0: \bar{\gamma}(w) \geqq \gamma^{*}(w)$. Let us denote by $H^{*}$ the solution operator of the following BVP

$$
L w+\gamma^{*}(w)=f \quad \text { in } \Omega, \quad B w=0 \quad \text { on } \partial \Omega .
$$

Since $H^{*}(0)=0$ and $H^{*}$ is strongly increasing we must have that $w_{0} \equiv H^{*}(-r) \ll 0$ in $C_{B}^{1}(\bar{\Omega})\left(u \ll v\right.$ means $v-u \in \operatorname{int}\left(P_{C_{B}{ }^{1}(\bar{\Omega})}\right), P_{C_{B}{ }^{1}(\bar{\Omega})}=$ positive cone in $\left.C_{B}^{1}(\bar{\Omega})\right)$. This implies the existence of a negative number $t^{\prime} \in \mathbb{R}$ such that $-t^{\prime} w_{0} \ll \phi-v$. We find

$$
\begin{aligned}
L\left(-t^{\prime} w_{0}\right)+\gamma^{*}\left(-t^{\prime} w_{0}\right) & =-t^{\prime}\left(L w_{0}+\gamma^{*}\left(w_{0}\right)\right)+\left(\gamma^{*}\left(-t^{\prime} w_{0}\right)+t^{\prime} \gamma^{*}\left(w_{0}\right)\right) \\
& =t^{\prime} r+\chi\left(\tilde{\gamma}\left(-t^{\prime} w_{0}\right)+t^{\prime} \tilde{\gamma}\left(w_{0}\right)\right) \text { in } \Omega \\
B w_{0} & =0 \text { on } \partial \Omega .
\end{aligned}
$$

Hence $\phi-v \gg-t^{\prime} w_{0}=H^{*}\left(t^{\prime} r+b\right)$ with $b=\chi\left(\tilde{\gamma}\left(-t^{\prime} w_{0}\right)+t^{\prime} \tilde{\gamma}\left(w_{0}\right)\right)$. We have for $t \leqq t^{\prime}$

$$
t^{\prime} r+b \geqq t r+b \geqq 2 t r-(t r-b)^{+} .
$$

The set $\left((t r-b)^{+}\right)_{t \leqq r^{\prime}}$ is $L^{\infty}$-bounded by $\|b\|_{\infty}$ and converges in $L^{p}$ to zero. By the 
continuity properties of $H^{*}$ we find $\delta>0$ such that for all $d \in L^{\infty}(\Omega),\|d\|_{\infty} \leqq\|b\|_{\infty}$ and $\|d\|_{L^{\mathrm{p}(\Omega)}} \leqq \delta$ we have

$$
\phi-v \gg H^{*}\left(t^{\prime} r+b+d\right) \text { in } C_{\mathrm{B}}^{1}(\bar{\Omega}) .
$$

For $t \leqq t^{\prime}$ small enough we have $\left\|(t r-b)^{+}\right\|_{L^{\mathfrak{b}}(\Omega)} \leqq \delta$. This implies for $d=(t r-b)^{+}$

$$
\begin{aligned}
\phi-v & \gg H^{*}\left(t^{\prime} r+b+(t r-b)^{+}\right) \\
& \geqq H^{*}\left(t r+b+(t r-b)^{+}\right) \\
& \geqq H^{*}\left(2 t r-(t r-b)^{+}+(t r-b)^{+}\right) \\
& =H^{*}(2 t r) .
\end{aligned}
$$

Let $T=2 t$. We obtain for $w^{*}=H^{*}(T r) \quad(\leqq 0)$

$$
\begin{aligned}
\operatorname{Tr} & =L w^{*}+\gamma^{*}\left(w^{*}\right) \\
& \leqq L w^{*}+\tilde{\gamma}\left(w^{*}\right) \\
& =L\left(w^{*}+v\right)+\gamma\left(w^{*}+v\right)-(L v+\gamma(v)) \\
& =L\left(w^{*}+v\right)+\gamma\left(w^{*}+v\right)-a \quad \text { in } \Omega .
\end{aligned}
$$

Hence

$$
\begin{aligned}
& L\left(w^{*}+v\right)+\gamma\left(w^{*}+v\right) \geqq a+\operatorname{Tr} \equiv L u+\gamma(u) \text { in } \Omega \\
& B\left(w^{*}+v\right)=B u=0 \quad \text { on } \partial \Omega .
\end{aligned}
$$

From this we deduce

$$
H(T r+a) \leqq w^{*}+v \ll \phi-v+v=\phi
$$

which completes the proof.

Remark. 3. If the hypotheses of part (b) are not satisfied, one can not expect that the solutions of $(*)$ are negative if $t$ tends to $-\infty$. Consider the following example:

Let $\Omega=(0,2)$. We study the following BVP

$$
-u^{\prime \prime}+q u+\tilde{\gamma}(x, u)=f+\operatorname{tr} \quad \text { in } \Omega, \quad u=0 \quad \text { on } \partial \Omega
$$

where $q=4 \exp (-2), \tilde{\gamma}: \bar{\Omega} \times \mathbb{R} \rightarrow \mathbb{R}$ is defined by

$$
\tilde{\gamma}(x, u)=-\exp (-2 u+4 x)+\exp (4 x)-q \min (u, 1-\ln (2)),
$$

and $f \in C(\bar{\Omega})$ by

$$
f(x)=\mid \begin{array}{ll}
\exp (4 x) & x \in[0,1] \\
0 & x \in[3 / 2,2] \\
\text { linear on } & (1,3 / 2)
\end{array}
$$

and $r \supsetneqq 0$ continuous with support in $(3 / 2,2)$.

One easily verifies that the operator $\gamma: C^{1}(\bar{\Omega}) \rightarrow L^{\infty}(\Omega): u \rightarrow \gamma(u): \gamma(u)(x)=$ $\tilde{\gamma}(x, u(x))$ is increasing, continuous and verifies the hypotheses of part (a). Let $v(x)=\ln (1-x)+2 x$ for $x \in[0,1)$. We have $v(0)=0,1-\ln (2)=v(1 / 2) \geqq v(x)$ for all $x \in[0,1)$ and $\lim _{x \rightarrow 1} v(x)=-\infty$. Denote the solution operator of $(* *)$ by $H$ and 
let $\boldsymbol{u}=\boldsymbol{H}(f+t r)$. We obtain on $[0,1-z]$ for some $z, 1 / 2>z>0$, small enough $-v^{\prime \prime}+q v+\tilde{\gamma}(x, v)=f=-u^{\prime \prime}+q u+\tilde{\gamma}(x, u)$ in $[0,1-z]$

$$
v(0)=u(0) \quad \text { and } \quad v(1-z) \leqq u(1-z) .
$$

By Lemma 1 we conclude that $v \leqq u$ on $[0,1-z]$. Hence

$$
0<1-\ln (2)=v(1 / 2) \leqq u(1 / 2)=H(f+t r)(1 / 2)
$$

for all $t \in \mathbb{R}$.

\section{Proofs of the main results}

Proof of Theorem 1. Define $\tilde{\gamma}: \bar{\Omega} \times \mathbb{R} \times \mathbb{R}^{n} \rightarrow \mathbb{R}$ by $\left(G_{s}=\partial G / \partial s\right)$

$$
\tilde{\gamma}(x, s, \xi)=\int_{0}^{s}\left(\operatorname{sign}\left(G_{s}(x, u, \xi)-\left|G_{s}(x, u, \xi)\right|\right) G_{s}(x, u, \xi)\right) d u+s .
$$

Then the maps $s \rightarrow \bar{\gamma}(x, s, \xi)$ and $s \rightarrow \tilde{\gamma}(x, s, \xi)+G(x, s, \xi)$ are strictly increasing for all fixed $(x, \xi) \in \bar{\Omega} \times \mathbb{R}^{n}$. Consider

$$
\begin{aligned}
L u+\tilde{\gamma}(x, u, D u) & =G(x, v, D u)+\tilde{\gamma}(x, v, D u)+\operatorname{tr} \quad \text { in } \Omega \\
B u & =0 \quad \text { on } \partial \Omega,
\end{aligned}
$$

where $v$ is a given function in $C(\bar{\Omega})$. We will show that (2) is for all $v$ uniquely solvable and that the solution operator $T: \mathbb{R} \times C(\bar{\Omega}) \rightarrow C_{B}^{1}(\bar{\Omega}):(t, v) \rightarrow u(t, v)$ is strongly increasing in both arguments and compact. Define $\tilde{\gamma}_{v}: \bar{\Omega} \times \mathbb{R} \times \mathbb{R}^{n} \rightarrow \mathbb{R}$ by

$$
\begin{aligned}
\tilde{\gamma}_{v}(x, s, \xi)= & \tilde{\gamma}(x, s, \xi)-G(x, v(x), \xi)-\tilde{\gamma}(x, v(x), \xi) \\
& +G(x, v(x), 0)+\tilde{\gamma}(x, v(x), 0) .
\end{aligned}
$$

Then $\tilde{\gamma}_{v}$ is locally uniformly lipschitz continuous in $s$ and $\xi$, satisfies $\left|\tilde{\gamma}_{v}(x, s, \xi)\right| \leqq$ $c_{v}(|s|)\left(1+|\xi|^{2}\right)$ for a suitable increasing function $c_{v}: \mathbb{R}^{+} \rightarrow \mathbb{R}^{+}$depending on $v$, and is increasing in $s$. Moreover we have

$$
\begin{aligned}
\tilde{\gamma}_{v}(x, s, \xi)-\tilde{\gamma}_{v}\left(x, s^{\prime}, \xi^{\prime}\right) & =\tilde{\gamma}_{v}(x, s, \xi)-\tilde{\gamma}_{v}\left(x, s^{\prime}, \xi\right)+\tilde{\gamma}_{v}\left(x, s^{\prime}, \xi\right)-\tilde{\gamma}_{v}\left(x, s^{\prime}, \xi^{\prime}\right) \\
& =b_{0}\left(s-s^{\prime}\right)+\sum_{i=1}^{n} b_{i}\left(\xi_{i}-\xi_{i}^{\prime}\right)
\end{aligned}
$$

where for $i=1 \ldots n$

$$
b_{i}(x)=\mid \begin{array}{ll}
\left(\tilde{\gamma}_{v}\left(x, s^{\prime}, \xi\right)-\tilde{\gamma}_{v}\left(x, s^{\prime}, \xi^{\prime}\right)\right)\left(\xi_{i}-\xi_{i}^{\prime}\right)^{-1} & \xi_{i} \neq \xi_{i}^{\prime} \\
0 & \text { otherwise }
\end{array}
$$

and

$$
b_{0}(x)=\mid \begin{array}{ll}
\left(\tilde{\gamma}_{v}(x, s, \xi)-\tilde{\gamma}_{v}\left(x, s^{\prime}, \xi\right)\right)\left(s-s^{\prime}\right)^{-1} & s \neq s^{\prime} \\
0 & \text { otherwise. }
\end{array}
$$

Since $\tilde{\gamma}_{v}$ is strictly increasing in $s$ we have $b_{0}(x)>0$ and by the lipschitz condition for $i=0 \ldots n: b_{i} \in L^{\infty}(\Omega)$. Let $\Omega^{*}=\Omega^{\circ}$. Since the mapping $(s, \xi) \rightarrow \tilde{\gamma}_{v}(x, s, \xi)$ is locally uniformly lipschitz continuous and satisfies for given $\tau \in \mathbb{R}^{+}$

$$
\left|\tilde{\gamma}_{v}(x, s, \xi)\right| \leqq c_{2}(\tau)(1+|s|+|\xi|) \text { for all }(x, s, \xi) \in \Omega^{*} \times \mathbb{R} \times \mathbb{R}^{n} \text { with } s \leqq \tau,
$$


we find for all given $w \in C^{1}(\bar{\Omega})$ a constant $M(w)$ such that

$$
\begin{array}{r}
\left|\tilde{\gamma}_{v}(x, s+w(x), \xi+D w(x))-\tilde{\gamma}_{v}(x, w(x), D w(x))\right| \leqq M(w)(|s|+|\xi|) \\
\text { for all }(x, s, \xi) \in \Omega^{*} \times \mathbb{R}^{-} \times \mathbb{R}^{n} .
\end{array}
$$

Let us define the operator $\gamma_{v}: C^{1}(\bar{\Omega}) \rightarrow L^{\infty}(\Omega)$ by $\gamma_{v}(u)=\tilde{\gamma}_{v}(., u, D u)$. By (3) we can find for all $w \in C^{1}(\bar{\Omega})$ a constant $M(w)>0$ such that

$$
\begin{aligned}
\left|\left(\gamma_{v}(u+w)-\gamma_{v}(w)\right)(x)\right| & \leqq M(w)(|u(x)|+|D u(x)|) \\
& \text { for all } x \in \Omega^{*} \text { and all } u \in C^{1}(\bar{\Omega}) \text { with } u \leqq 0 .
\end{aligned}
$$

The preceding discussion of $\tilde{\gamma}_{v}$ implies that $\gamma_{v}$ satisfies the hypotheses of the proposition.

This implies that (2) is uniquely solvable because it is equivalent to

$$
L u+\gamma_{v}(u)=G(., v, 0)+\tilde{\gamma}_{v}(., v, 0)+\operatorname{tr} \text { in } \Omega \quad B u=0 \quad \text { on } \partial \Omega .
$$

Let $T: \mathbb{P} \times C(\bar{\Omega}) \rightarrow C_{B}^{1}(\bar{\Omega}):(t, v) \rightarrow u(t, v)$ be the solution operator of (4). Using Lemma 2 and proceeding as in the proof of the proposition, part (a), we deduce that $T$ is strongly increasing and compact. The solution $u$ of

$$
L u+\gamma_{0}(u)=G(., 0,0)+\tilde{\gamma}_{0}(., 0,0)+\operatorname{tr} \text { in } \Omega \quad B u=0 \text { on } \partial \Omega
$$

is by definition $T(t, 0)$. Applying now the proposition, we conclude for $t^{*} \leqq 0$ small enough $T\left(t^{*}, 0\right) \ll 0$. From results in [1] (Proposition 2.13) we obtain because of the asymptotic behaviour of $G$ as $s \rightarrow-\infty$, that there exists a strict subsolution $\bar{u} \leqq 0$ of $\left(\mathrm{P}_{t^{*}}\right)$ which implies $T\left(t^{*}, \bar{u}\right) \gg \bar{u}$ in $C_{B}^{1}(\bar{\Omega})$. Since $T\left(t^{*},.\right)$ is strongly increasing, it maps $V \equiv[\bar{u}, 0]_{C_{B}{ }^{1}(\bar{\Omega})} \rightarrow$ int $(V)$. Moreover $V$ is bounded in $C(\bar{\Omega})$ which implies that $T\left(t^{*}, V\right)$ is bounded in $C_{B}^{1}(\bar{\Omega})$. Hence, by the compactness of $T\left(t^{*},.\right): C(\bar{\Omega}) \rightarrow C(\bar{\Omega})$, we find a fixed point in int $(V)$ by Schauder's fixed point theorem. Let $t_{0} \equiv \sup \left\{t \in \mathbb{R} \mid\left(\mathrm{P}_{t}\right)\right.$ is solvable $\}$. If $\left(\mathrm{P}_{t}\right)$ has a solution $u_{t}$ for some $t$ we infer that $u_{t}$ is a strict supersolution for all $t^{\prime}<t$. As above we find a strict subsolution $\bar{u} \leqq u_{t}$, which implies the existence of a solution $u_{t^{\prime}} \in \operatorname{int}\left(\left[\bar{u}, u_{t}\right]_{C_{B}{ }^{1}(\bar{\Omega})}\right)$ of $\left(\mathrm{P}_{t}\right)$. By the asymptotic behaviour of $G$ as $s \rightarrow+\infty$, one can show that $t_{0}<+\infty$ (see [1, proof of Theorem 3.4, p. 636]).

Proof of Theorem 2. Let $T$ be as in Theorem 1 and choose $t<t_{0}$. As we have seen in the proof above there exist strict sub- and supersolutions of $\left(P_{t}\right): \bar{u} \leqq \hat{u}$. Denote the order interval $[\bar{u}, \hat{u}]_{C_{\mathrm{B}}{ }^{1}(\bar{\Omega})}$ by $A$. Since $T=T(t,$.$) maps A \rightarrow \operatorname{int}(A)$ we find that the fixed points of $T$ in $A$ are in int $(A)$. We may assume that there is only one fixed point $u \in A$ (otherwise we are done). Finally we find for some $\varepsilon>0$ such that $u+\varepsilon \mathbb{B} \subset \operatorname{int}(A)$, where $\mathbb{B}$ denotes the open unit ball in $C_{B}^{1}(\bar{\Omega})$, making use of the standard properties of the Leray-Schauder-Degree and the fixed point index

$$
\begin{aligned}
\operatorname{deg}(I-T, u+\varepsilon \mathbb{B}, 0) & =i\left(T, u+\varepsilon \mathbb{B}, C_{B}^{1}(\bar{\Omega})\right)=i(T, u+\varepsilon \mathbb{B}, A) \\
& =i(T, A, A)=1 .
\end{aligned}
$$

For a solution $u$ of $T(\bar{t}, u)=u, \bar{t} \in\left[t, t_{0}+1\right] \equiv \Gamma$, we have $u(x) \leqq M(\Gamma)$. On the 
other hand solutions are bounded from below by

$$
\begin{aligned}
L u & =G(x, u, D u)+t r \\
& \geqq G^{*}(x, u)+t r \\
& \geqq\left(\lambda_{1}-\bar{\varepsilon}\right) u+h \quad \text { in } \Omega \\
B u & =0 \text { on } \partial \Omega
\end{aligned}
$$

for some $\bar{\varepsilon}>0$ small enough and a suitable constant $h$ depending only on $\bar{\varepsilon}$ and $\Gamma$. Hence $u \geqq K(h)$, where $K$ denotes the positive solution operator of

$$
L u-\left(\lambda_{1}-\bar{\varepsilon}\right) u=g \quad \text { in } \Omega, \quad B u=0 \quad \text { on } \partial \Omega .
$$

Since the solutions of $\left(\mathbf{P}_{\bar{t}}\right), \bar{t} \in \Gamma$, are contained in a $C(\bar{\Omega})$-bounded set, they must be bounded in $C_{B}^{1}(\bar{\Omega})$ by a constant $k-1$. We deduce, since we may assume $u+\varepsilon \mathbb{B} \subset k \mathbb{B}$, making use of the additivity, homotopy and excision properties of the Leray-Schauder-Degree

$$
\begin{aligned}
\operatorname{deg}(I-T, k \mathbb{B} \backslash u+\varepsilon \mathbb{B}, 0) & =\operatorname{deg}(I-T, k \mathbb{B}, 0)-1 \\
& =\operatorname{deg}\left(I-T\left(t_{0}+1, .\right), k \mathbb{B}, 0\right)-1 \\
& =-1
\end{aligned}
$$

which implies the existence of a second solution. To study $\left(\mathbf{P}_{t_{0}}\right)$ choose a sequence $\left(t_{n}\right), t_{n}<t_{0}, t_{n} \rightarrow t_{0}$. One can show as before that the sequence $\left(u_{n}\right)$ of solutions $u_{n}$ of $\left(\mathbf{P}_{t_{n}}\right)$ is relatively compact in $C_{B}^{1}(\bar{\Omega})$. Hence we may assume (for a subsequence) $u_{n} \rightarrow u$ strongly in $C_{\mathbf{B}}^{1}(\bar{\Omega})$. Taking the limit for $T\left(t_{n}, u_{n}\right)=u_{n}$ we find $T\left(t_{0}, u\right)=u$ which completes the proof.

Proof of the Corollary. It is enough to show that for all bounded intervals $\Gamma \subset \mathbb{R}$ there exists a constant $M=M(\Gamma)$ such that a solution $u$ of $\left(\mathrm{P}_{t}\right), t \in \Gamma$, satisfies $u(x) \leqq M$ for all $x \in \bar{\Omega}$. As in the proof of Theorem 2 one can show the existence of a constant $M^{*}: u$ is solution of $\left(\mathrm{P}_{t}\right), t \in \Gamma$, then $u(x) \geqq-M^{*}$ for all $x \in \bar{\Omega}$. Assume there exist sequences $\left(t_{n}\right)$ and $\left(u_{n}\right) \subset W^{2, \mathrm{p}}(\Omega)$ with $\left\|u_{n}\right\|_{\infty} \rightarrow \infty$ verifying

$$
L u_{n}=G\left(x, u_{n}, D u_{n}\right)+t_{n} r \quad \text { in } \Omega, \quad B u_{n}=0 \quad \text { on } \partial \Omega .
$$

Since $\left\|u_{n}\right\|_{\infty} \rightarrow \infty$ we have $\left\|u_{n}\right\|=\left\|u_{n}\right\|_{C_{B}^{1}(\bar{\Omega})} \rightarrow \infty$. For $w_{n}=u_{n} /\left\|u_{n}\right\|$ we obtain

$$
\begin{aligned}
L w_{n} & =\left(G\left(x, u_{n}, D u_{n}\right)+t_{n} r\right) /\left\|u_{n}\right\| \\
& \geqq\left(G^{*}\left(x, u_{n}\right)+t_{n} r\right) /\left\|u_{n}\right\| \\
& \geqq\left(\lambda_{1}+\varepsilon\right) w_{n}-\eta /\left\|u_{n}\right\|
\end{aligned}
$$

for suitable constants $\varepsilon>0$ and $\eta>0$ depending on $\Gamma$. By the boundedness of the set $\left(\left(G\left(x, u_{n}, D u_{n}\right)+t_{n} r\right) /\left\|u_{n}\right\|\right)$ (Condition $\left.(G 3)\right)$ in $C(\bar{\Omega})$ we infer the relative compactness of $\left(w_{n}\right)$ in $C_{B}^{1}(\bar{\Omega})$. Hence we may assume $w_{n} \rightarrow w$ in $C_{B}^{1}(\bar{\Omega})$ for some $w \in C_{B}^{1}(\bar{\Omega})$ having $\|w\|=1$. Since $\left(u_{n}\right)$ is bounded from below we have $w \supsetneqq 0$. Thus we deduce from (11) taking the limit

$$
\left(\lambda_{1}+\varepsilon\right)^{-1} w-\bar{K} w=y \geqq 0
$$

where $\bar{K}$ denotes the solution operator of the linear BVP $L u=g$ in $\Omega, B u=0$ on $\partial \Omega$, where $\mathrm{g}$ is a given function in $L^{p}(\Omega)$. Since $\bar{K}: C_{\mathbf{B}}^{1}(\bar{\Omega}) \rightarrow C_{B}^{1}(\bar{\Omega})$ is a strongly 
positive compact endomorphism and the spectral radius is $r(\overline{\bar{K}})=\lambda_{1}^{-1}$ we conclude, observing that $\left(\lambda_{1}+\varepsilon\right)^{-1}<r(\bar{K})$, that equation (7) cannot have a positive solution (see [3, Theorem 3.2]). This contradiction proves the corollary.

\section{References}

1 J. L. Kazdan and R. J. Kramer. Invariant criteria for existence of solutions to second order quasilinear elliptic equations. Comm. Pure Appl. Math. 31 (1978), 619-645.

2 P. Hess. On a nonlinear elliptic boundary value problem of the Ambrosetti-Prodi type. Boll. Un. Mat. Ital. 17 (1980), 187-192.

3 H. Amann. Fixed point equations and nonlinear eigenvalue problems in ordered Banach spaces. SIAM Rev. 18 (1976), 620-709.

4 H. Amann and M. Crandall. On some existence theorem for semilinear elliptic equations. Indiana Univ. Math. J. 27 (1978), 779-790.

5 J. L. Kazdan and F. W. Warner. Remarks on some quasilinear elliptic equations. Comm. Pure Appl. Math. 28 (1975), 567-597.

6 J. M. Bony. Principe du maximum dans les espace de Sobolev. C.R. Acad. Sci. Paris Ser. A 265 (1967), 333-336.

7 H. Amann and P. Hess. A multiplicity result for a class of elliptic boundary value problems. Proc. Roy. Soc. Edinburgh Sect. A 84 (1979), 145-151.

8 E. N. Dancer. On the ranges of certain weakly nonlinear elliptic partial differential equations. $J$. Math. Pures Appl. 57 (1978), 351-366.

(Issued 11 March 1981) 